KSUPT-03/4 August 2003

\title{
Non-Gaussian Error Distribution of Hubble Constant Measurements
}

\author{
Gang Chen ${ }^{1}$, J. Richard Gott, III $^{2}$, and Bharat Ratra ${ }^{1}$
}

\begin{abstract}
We construct the error distribution of Hubble constant $\left(H_{0}\right)$ measurements from Huchra's compilation of 461 measurements of $H_{0}$ and the WMAP experiment central value $H_{0}=71 \mathrm{~km} \mathrm{~s}^{-1} \mathrm{Mpc}^{-1}$. This error distribution is non-Gaussian, with significantly larger probability in the tails of the distribution than predicted by a Gaussian distribution. The $95.4 \%$ confidence limits are $7.0 \sigma$ in terms of the quoted errors. It is remarkably well described by either a widened $n=2$ Student's $t$ distribution or a widened double exponential distribution. These conclusions are unchanged if we use instead the central value $H_{0}=67 \mathrm{~km} \mathrm{~s}^{-1} \mathrm{Mpc}^{-1}$ found from a median statistics analysis of a major subset of $H_{0}$ measurements used here.
\end{abstract}

Subject headings: cosmology: observation — methods: statistical — methods: data analysis - cosmology: distance scale — large-scale structure of the universe

\section{Introduction}

Measurements of the Hubble constant are a unique data set for statistical analysis for two reasons. First, Huchra's compilation ${ }^{3}$ with over 400 measurements is one of the largest collection of measurements of a single quantity. Second, the Hubble constant is now one of the more precisely determined cosmological parameters (see, e.g., Freedman et al. 2001; Bennett et al. 2003).

It is also of great interest to understand how well the Hubble constant has been measured, both because it is an important cosmological parameter and because of the role it plays in various cosmological tests, most importantly the expansion time test (see, e.g., Peebles \& Ratra 2003 for a review).

Assuming a value for the Hubble constant — in the body of this paper we work with $H_{0}=$ $71 \mathrm{~km} \mathrm{~s}^{-1} \mathrm{Mpc}^{-1}$, the central value from the combined WMAP and other data analysis of Spergel

\footnotetext{
${ }^{1}$ Department of Physics, Kansas State University, 116 Cardwell Hall, Manhattan, KS 66506.

${ }^{2}$ Princeton University Observatory, Peyton Hall, Princeton, NJ 08544.

${ }^{3}$ See cfa-www.harvard.edu/ huchra/.
} 
et al. $(2003)^{4}$ - one may use Huchra's compilation of $H_{0 i}^{+\sigma_{i}^{\mathrm{u}}}$ (where $\sigma_{i}^{\mathrm{u}}$ and $\sigma_{i}^{\mathrm{l}}$ are the upper and lower one standard deviation error bars) to construct the distribution of errors of the Hubble constant measurements. This is a plot of the number of measurements as a function of the number of standard deviations $\left(N_{\sigma}\right)$ the measurement deviates from the actual value $H_{0}$. Here

$$
N_{\sigma}=\frac{H_{0 i}-H_{0}}{\sigma_{i}^{\mathrm{u}}}
$$

when $H_{0 i}<H_{0}$ and

$$
N_{\sigma}=\frac{H_{0 i}-H_{0}}{\sigma_{i}^{1}}
$$

when $H_{0 i}>H_{0}$.

In our analysis here we use measurements from Huchra's compilation up to and including measurement 2003.239. Deleting the four entries from 1924 and 1925 that lack actual estimates of $H_{0}$, we use 461 published estimates of $H_{0}$ in our analysis here, $40 \%$ more than the 331 used in the analysis of Gott et al. (2001). Observers often note that there could be unknown systematic errors, however authors' quoted errors have been used to evaluate the accuracy of $H_{0}$ estimates and so it is important to understand the quoted error distribution.

In $\S 2$ we describe our analysis of this collection of 461 measurements, assuming that $H_{0}=71$ $\mathrm{km} \mathrm{s}^{-1} \mathrm{Mpc}^{-1}$, the central value from the combined WMAP and other data analysis of Bennett et al. (2003) and Spergel et al. (2003). For comparison, to show that the results are robust to small changes in the true value of $H_{0}$, summary results from an analysis based on the central value of $H_{0}=67 \mathrm{~km} \mathrm{~s}^{-1} \mathrm{Mpc}^{-1}$ from the Gott et al. (2001) median statistics study are presented in the Appendix. We conclude in $\S 3$.

\section{Analysis}

Figure 1 shows the distribution of deviations of the 461 measurements from the central WMAP value of $H_{0}=71 \mathrm{~km} \mathrm{~s}^{-1} \mathrm{Mpc}^{-1}$, in units of the quoted standard deviation of the measurement. This is the error distribution of the $H_{0}$ measurements; the left panel shows the signed error distribution and the right panel shows the absolute magnitude of the errors (the distribution in the right panel is symmetric about $\left|N_{\sigma}\right|=0$ ). These error distributions have significant tails: there are numerous measurements 5 and even 10 standard deviations away. More precisely, in the signed error distribution of Fig. $1 a 68.3 \%$ and $95.4 \%$ of the probability lies in the range $-2.4 \leq N_{\sigma} \leq 1.1$ and $-7.0 \leq N_{\sigma} \leq 6.7$, respectively, and for the absolute magnitude error distribution of Fig. $1 b$ the

\footnotetext{
${ }^{4}$ In the Appendix we summarize results from a similar analysis based on the central value of $H_{0}=67 \mathrm{~km} \mathrm{~s}$ $\mathrm{Mpc}^{-1}$ from the Gott et al. (2001) median statistics analysis of a major, earlier subset of the $H_{0}$ measurements considered here, showing that our conclusions are not sensitive to the precise central value of $H_{0}$ assumed in the estimated $10 \%$ (two standard deviation) range now under discussion (see, e.g., Gott et al. 2001).
} 
corresponding limits are $\left|N_{\sigma}\right| \leq 1.9$ and $\left|N_{\sigma}\right| \leq 7.0$, respectively. An alternative characterization of the tails of this distribution is provided by the fraction of data within the $\left|N_{\sigma}\right|=1$ and 2 ranges, which for the distribution shown in Fig. $1 b$ is $48 \%$ and $69 \%$, respectively. These are impressively high (nearly half the observed values are within one standard deviation of $71 \mathrm{~km} \mathrm{~s}^{-1} \mathrm{Mpc}^{-1}$ ) but still clearly at odds with what is expected for a Gaussian distribution.

It is of interest to quantify how well the data of Fig. 1 are fit by various simple distribution functions, and to determine the parameters of these functions that result in the best fit to the data. To do this we proceed as follows. For our purposes it is useful to maximize the number of data points in each bin as well as the number of bins. This is perhaps best accomplished by using 21 bins (close to the square root of 461), labelled by integer $j$ that runs from 1 to 21 , and adjusting the widths of the bins, $\Delta\left|N_{\sigma}\right|_{j}$, to ensure equal expected probability (for the assumed distribution function) in each bin. Thus, for an assumed distribution (such as a Gaussian) we construct 21 bins such that the expected number of data points in each bin would be 21.95. Then we compare with the number of data points observed in each of the 21 bins and do a $\chi^{2}$ analysis, as discussed in the next paragraph. (Since the number expected in each bin is large compared to unity, a $\chi^{2}$ analysis is justified.) With this prescription, the data binning depends on the assumed probability distribution function, $P\left(\left|N_{\sigma}\right|\right)$ (in this paper we present results only from the fit to the symmetric absolute error distribution, e.g., that in Fig. 1b).

To estimate goodness of fit we use the assumed probability distribution function to compute the expected number of measurements in each bin $j, N P\left(\left|N_{\sigma}\right|_{j}\right)$, where $N=461$ is the total number of measurements. Since there are a finite number of measurements in each bin, they should be Poisson distributed with mean value $N P\left(\left|N_{\sigma}\right|_{j}\right)$ for the $j^{\text {th }}$ bin. For the Poisson distribution the variance $\sigma_{j}^{2}$ is equal to the mean hence the total $\chi^{2}$ is

$$
\chi^{2}=\sum_{j=1}^{21} \frac{\left[M\left(\left|N_{\sigma}\right|_{j}\right)-N P\left(\left|N_{\sigma}\right|_{j}\right)\right]^{2}}{N P\left(\left|N_{\sigma}\right|_{j}\right)},
$$

where $M\left(\left|N_{\sigma}\right|_{j}\right)$ is the observed number of measurements in each bin. We shall tabulate the reduced $\chi^{2}, \chi_{\nu}^{2}=\chi^{2} / \nu$, where $\nu$ is the number of degrees of freedom, i.e., the number of bins (21) less the number of constraints and fitting parameters. Given $\chi_{\nu}^{2}$ and $\nu$ one may compute the probability that the assumed distribution well describes the spread of the measurements. In the computation of this probability we assume that the bins are uncorrelated, which is not necessarily true (since lower rungs of the distance ladder introduce correlations in subsets of the measurements). It is therefore wise to place quantitative emphasis on just the $\chi_{\nu}^{2}$ values and use the corresponding probabilities as simply a qualitative indicator of goodness of fit.

We consider four probability distribution functions and as mentioned above focus on the absolute magnitude error distribution, as in Fig. $1 b$, so all distributions we consider will be centered at $\left|N_{\sigma}\right|=0$. One constraint that must be satisfied is that the total number of measurements must sum to 461. Since we consider 21 bins and normalize to fit the total number of measurements, a probability distribution function with no free parameters will have $\nu=20$ degrees of freedom. 
Even though we have noted the existence of extended tails in the error distributions of Fig. 1, it is natural - perhaps pavlovian — to first consider the Gaussian distribution, initially with width chosen so that $\left|N_{\sigma}\right|=1$ corresponds to one standard deviation, and then with a scale factor to vary the width of the distribution. That is, we take as probability distribution function the Gaussian expression

$$
P\left(\left|N_{\sigma}\right|\right)=\frac{1}{\sqrt{2 \pi}} \exp \left[-\left|N_{\sigma}\right|^{2} / 2\right]
$$

for the case where $\left|N_{\sigma}\right|=1$ is equivalent to one standard deviation, and then consider the function $P\left(\left|N_{\sigma}\right| / S\right)$ where $S$ is a scale factor that is adjusted to minimize $\chi^{2}$. (We allow $S$ to vary over the range 0.5 to 3 in steps of 0.1 when computing $\chi^{2}$.) In the first case there are no additional free parameters so $\nu=20$; the scale factor $S$ is an additional free parameter in the second case so here we have $\nu=19$ degrees of freedom. Figure 2 shows the measurement error histograms and the best-fit Gaussians, both normalized to unit area. Numerical values are listed in Table 1. These show that if $H_{0}=71 \mathrm{~km} \mathrm{~s}^{-1} \mathrm{Mpc}^{-1}$ then the measurement error distributions are extremely poorly fit by a Gaussian, even if the Gaussian width is allowed to be a free parameter. Interestingly, if the width is allowed to float while minimizing $\chi^{2}$ it favors 1.8, i.e., the assumed distribution favors identifying $\left|N_{\sigma}\right|=1.8$ with one standard deviation, almost double the value one would naively infer from the measurement errors, thus perhaps indicating that in this Gaussian case it might not be unreasonable to roughly double the quoted error bars, consistent with our earlier discussion of extended tails. This situation might profitably be contrasted with what happened in the early days of cosmic microwave background spatial anisotropy measurements, where a number of models fit the measurements extremely well, perhaps indicating that the error bars had been over estimated (Ganga, Ratra, \& Sugiyama 1996). In any case, it is very unlikely that the $H_{0}$ measurement errors are described by a Gaussian distribution. (Note that the probability is a little higher for the $H_{0}=$ $67 \mathrm{~km} \mathrm{~s}^{-1} \mathrm{Mpc}^{-1}$ case, but even here a Gaussian distribution is a very poor fit.)

The fact that the error distribution of Hubble constant measurements is non-Gaussian does not necessarily imply an underlying non-Gaussianity in the measurement errors. Rather, the distribution tells us something about the observers ability to correctly estimate systematic and statistical uncertainties.

Figure 2 indicates that the distribution of Hubble constant measurement errors has a more extended tail than is predicted by a Gaussian probability distribution. Perhaps the most well-known distribution with an extended tail is the Cauchy, or Lorentzian, or Breit-Wigner distribution,

$$
P\left(\left|N_{\sigma}\right|\right)=\frac{1}{\pi} \frac{1}{1+\left|N_{\sigma}\right|^{2}}
$$

we also consider the case $P\left(\left|N_{\sigma}\right| / S\right)$ where the scale factor $S$ is allowed to vary while $\chi^{2}$ is minimized. Figure 3 shows the data and best-fit Cauchy distributions, and numerical values are listed in Table 1. Unlike the Gaussian case, the Cauchy distribution can not be rejected; it is acceptable at 9.9 $\%$ or $8.7 \%$ depending on whether $S$ is fixed to unity or allowed to vary (and it does significantly better at $H_{0}=67 \mathrm{~km} \mathrm{~s}^{-1} \mathrm{Mpc}^{-1}$ ). However, it is clear from Fig. 3 that the Cauchy distribution 
has greater probability in the extended tails than does the Hubble constant measurements error distribution. The Cauchy distribution has a similar central peak, with a $50 \%$ chance that $\left|N_{\sigma}\right|<1$, but a $95.4 \%$ chance that $\left|N_{\sigma}\right|<13.8$ instead of 7.0 as observed. It would therefore be beneficial to search for a distribution that has broader tails than the Gaussian one but narrower than the Cauchy case.

A Cauchy distribution with $S=1$ would result if the errors were Gaussian distributed, observers took measurements free of systematic errors, divide their data into two parts, used each half to produce two independent estimates of the Hubble constant, $H_{1}$ and $H_{2}$, and produced a mean estimate $H_{\mathrm{m}}=\left(H_{1}+H_{2}\right) / 2$ with an error estimate (standard deviation of the mean) of $\sigma_{\mathrm{m}}=\left|H_{1}-H_{2}\right| / 2$. If $H_{1}$ and $H_{2}$ are drawn from an underlying Gaussian distribution centered on the true value $H_{\mathrm{t}}$ then $\left(H_{\mathrm{m}}-H_{\mathrm{t}}\right) / \sigma_{\mathrm{m}}$ is distributed like a Cauchy distribution with $S=1$. That gives a $50 \%$ chance that $H_{\mathrm{m}}$ is within $1 \sigma_{\mathrm{m}}$ of the true value and a $95 \%$ chance that $H_{\mathrm{m}}$ is within $12.7 \sigma_{\mathrm{m}}$ of the true value. The large tails result because in a Gaussian distribution there is an appreciable chance that $\left|H_{1}-H_{2}\right| / 2$ will be significantly less than the true sigma for the distribution. In this scenario the observer is really using the self-consistency of her observations to set the error bars. If one measures the distance to two galaxies using cepheids, and gets two values for the Hubble constant that are close to each other, one may well be tempted to think that one's method has the high degree of accuracy implied by the observed value of $\sigma_{\mathrm{m}}=\left|H_{1}-H_{2}\right| / 2$. Indeed, if one estimated the errors by other means (estimated uncertainties in measuring the observed quantities required to measure the Hubble constant, along with standard propagation of errors) and one got an error significantly larger than $\left|H_{1}-H_{2}\right| / 2$ then one might be suspicious that one should be so lucky as to obtain such a small value of $\left|H_{1}-H_{2}\right| / 2$. Yet rarely, such lucky coincidences do occur and it is precisely these cases that cause the large tails in the Cauchy distribution. The Cauchy distribution with $S=1$ is acceptable at $9.9 \%$, but not a good fit and there are other distributions that are better fits. As we have noted, a Cauchy distribution with $S=1$ would result from a true Gaussian distribution if the observer divided his data into two parts, used the data itself to set error bars, and made the mistake of assuming the errors should be distributed according to a Gaussian distribution rather than the Student $t$ distribution (which for the case of two data points is the $n=1$ Student $t$ distribution, or the Cauchy distribution). This prompts us to investigate the general Student $t$ distributions.

Student's $t$ distribution is

$$
P_{n}\left(\left|N_{\sigma}\right|\right)=\frac{\Gamma[(n+1) / 2]}{\sqrt{\pi n} \Gamma(n / 2)} \frac{1}{\left(1+\left|N_{\sigma}\right|^{2} / n\right)^{(n+1) / 2}},
$$

where $n$ is positive and $\Gamma$ the Gamma function. We also consider the distribution $P_{n}\left(\left|N_{\sigma}\right| / S\right)$ where the scale factor $S$ is allowed to vary while $\chi^{2}$ is minimized. When $n \rightarrow \infty$ Student's $t$ distribution becomes the Gaussian distribution and for $n=1$ it is the Cauchy distribution. For $1<n<\infty$ Student's $t$ distribution has narrower tails than the Cauchy case but broader ones than the Gaussian distribution, just as wanted. We have fit Student's $t$ distribution to the $H_{0}$ measurement errors data while allowing $n$ to take on integer values between 2 and 6 (and sometimes going up to 30), 
so in this case we have one additional parameter and hence one less degree of freedom. We find $n=2$ always minimizes the value of $\chi^{2}$ and so show this case in Fig. 4 and Table 1. From Table 1 we see that if the scale factor $S$ is held at unity Student's $t$ distribution is an unlikely fit to the data, especially if $H_{0}=71 \mathrm{~km} \mathrm{~s}^{-1} \mathrm{Mpc}^{-1}$. However, if $S$ is allowed to vary as $\chi^{2}$ is minimized, Student's $t$ distribution with $n=2$ is an excellent fit to the $H_{0}$ measurements error distribution, and Fig. $4 b$ shows that there is very good agreement between the expected and measured counts in the last bin.

The final probability density distribution we consider is the double exponential or Laplace distribution,

$$
P\left(\left|N_{\sigma}\right|\right)=\frac{1}{2} e^{-\left|N_{\sigma}\right|} .
$$

This falls off less rapidly than the Gaussian distribution but faster than the Cauchy distribution. The sample median is the best estimator for the mean of this distribution (Eadie et al. 1971). The results of the fit are shown in Fig. 5 and listed in Table 1. As in the case for Student's $t$ distribution with $n=2$, when $S$ is held fixed at unity the double exponential is an unacceptable fit to the $H_{0}$ measurements error distribution but when $S$ is allowed to vary it is an excellent fit to the data.

In the first paragraph of this section we noted that in Fig. $1 b 68.3 \%$ and $95.4 \%$ of the probability lies in the range $\left|N_{\sigma}\right| \leq 1.9$ and $\left|N_{\sigma}\right| \leq 7.0$, respectively, and the $\left|N_{\sigma}\right| \leq 1$ and $\left|N_{\sigma}\right| \leq 2$ ranges include $48 \%$ and $69 \%$ of the data points, respectively. (See the Appendix for the corresponding numbers for the $H_{0}=67 \mathrm{~km} \mathrm{~s}^{-1} \mathrm{Mpc}^{-1}$ case.) Tables 2 and 3 show the related limits for the various probability density distributions we consider in this paper. These numerical values provide another indication of the non-Gaussianity of the Hubble constant measurement error distribution.

\section{Conclusion}

Our analysis of a perhaps unique (because of its size) data set, the measurement errors of all available estimates of the Hubble constant, makes for some interesting conclusions. If all observers have done perfect jobs at estimating their errors and the true errors were Gaussian, as might be expected, then the distributions in Fig. 1 should be Gaussian with standard deviation of unity.

First, and perhaps not totally unexpectedly, the errors in the Hubble constant are not Gaussianly distributed, even if the scale factor $S$ is allowed to vary when minimizing $\chi^{2}$. At the minimum value of $\chi^{2}, S \sim 2$, suggesting it might be reasonable to roughly double the magnitude of $H_{0}$ measurement error bars. Early observers using inferior equipment or techniques would have larger errors, but knowing that their methods were uncertain should have established larger error bars. As methods improved the measurements become more accurate but the stated error bars become smaller. Early or late observers are at no relative disadvantage relative to others. Indeed, each observer has freedom to state her error bars and has a priori an equal chance of having the true value occur within one standard deviation of their result. Over-optimism would produce error bars that 
were too small while over-conservatism would produce error bars that were too large. Which occurs in practice? The results here suggest that astronomers were over-optimistic by almost a factor of 2. Why? In some case there were systematic errors of which the observers were simply unaware (such as mistaking HII regions for bright stars). In other cases, standard candles were not as standard as imagined, leaving some steps in the distance ladder wrong by more than people thought. Also, using self consistency in the data as a check on the errors can lead to large tails because it occasionally induces one to be over-optimistic (the Student $t$ effect). And the real data may have non-Gaussian tails (say in the luminosity of standard candles). In general over-conservatism (the urge to be right) always competes with over-optimism (the urge to have more interesting limits). In the case of the Hubble constant astronomers were over-optimistic. In a history-of-science context, it might be of interest to more closely examine the most deviant measurements of Fig. 1, those that have $\left|N_{\sigma}\right|$ larger than say 7 , to understand why these are so deviant, but this is not our purpose here.

The Hubble constant measurement history suggests that to be really sure (95.4\%) you have to go to $7 \sigma$. This may explain why some people are cautious upon hearing of a three standard deviation result. It's not that they believe the errors but want to be more sure than $99.7 \%$. It's that they suspect there is a large chance $(\sim 50 \%)$ that the error bars may have been underestimated by a factor of 2 or 3 and the chance it is really correct is consequently really significantly less than $99.7 \%$.

Second, an $n=2$ Student's $t$ distribution, with $S \sim 1.2-1.3$, or a double exponential distribution, with $S \sim 1.5-1.6$, are excellent fits to the $H_{0}$ measurement errors distribution, with $H_{0}=67 \mathrm{~km} \mathrm{~s}^{-1} \mathrm{Mpc}^{-1}$ having a somewhat higher probability than $H_{0}=71 \mathrm{~km} \mathrm{~s}^{-1} \mathrm{Mpc}^{-1}$.

The Hubble constant measurement history gives an interesting example where we can access how trustworthy quoted errors might be in fundamental measurements. It would be interesting to study comparative examples from other fields. In particular, it would be interesting to know whether the $n=2$ Student $t$ distribution or the double exponential distribution also provides a good description of the measurement errors of other quantities.

We are grateful to J. Huchra for the compilation of $H_{0}$ measurements and acknowledge useful discussions with A. Kosowsky. GC and BR acknowledge support from NSF CAREER grant AST9875031 and DOE EPSCoR grant DE-FG02-00ER45824. JRG acknowledges support from NSF grant AST-9900772.

\section{A. $H_{0}=67 \mathrm{~km} \mathrm{~s}^{-1} \mathrm{Mpc}^{-1}$}

In the main body of the paper we assumed $H_{0}=71 \mathrm{~km} \mathrm{~s}^{-1} \mathrm{Mpc}^{-1}$, the central value from the WMAP analysis. The WMAP $H_{0}$ error bars are ${ }_{-3}^{+4} \mathrm{~km} \mathrm{~s}^{-1} \mathrm{Mpc}^{-1}$ (Bennett et al. 2003). $H_{0}$ is pinned down to only about $10 \%$ at two standard deviations (Gott et al. 2001) so it is reasonable 
to find out how our conclusions depend on the value of $H_{0}$. In this Appendix we use $H_{0}=67$ $\mathrm{km} \mathrm{s}^{-1} \mathrm{Mpc}^{-1}$, the central value from the Gott et al. (2001) median statistics analysis of a subset (331 measurements prior to mid 1999) of the 461 measurements used here. ${ }^{5}$ Figure 6 shows the $H_{0}$ measurements error distribution for the case when $H_{0}=67 \mathrm{~km} \mathrm{~s}^{-1} \mathrm{Mpc}^{-1}$. This distribution has a somewhat less prominent central peak than the $H_{0}=71 \mathrm{~km} \mathrm{~s}^{-1} \mathrm{Mpc}^{-1}$ case. In the signed error distribution of Fig. $6 a 68.3 \%$ and $95.4 \%$ of the probability lies in the range $-1.8 \leq N_{\sigma} \leq 1.7$ and $-5.7 \leq N_{\sigma} \leq 7.9$, respectively, while for the absolute magnitude error distribution of Fig. $6 b$ the corresponding limits are $\left|N_{\sigma}\right| \leq 1.7$ and $\left|N_{\sigma}\right| \leq 7.5$, respectively. In Fig. $6 b$ the $\left|N_{\sigma}\right| \leq 1$ and $\left|N_{\sigma}\right| \leq 2$ ranges include $51 \%$ and $72 \%$ of the data points, respectively. Again, these are at odds with what is expected for a Gaussian distribution.

Table 1 also lists the numerical fitting results for the $H_{0}=67 \mathrm{~km} \mathrm{~s}^{-1} \mathrm{Mpc}^{-1}$ case. As in the case when $H_{0}=71 \mathrm{~km} \mathrm{~s}^{-1} \mathrm{Mpc}^{-1}$, here again the $n=2$ Student's $t$ distribution and the double exponential distribution provide excellent fits to the $H_{0}$ error histogram when the scale factor $S$ is allowed to vary when minimizing $\chi^{2}$. These two distributions are shown in Figs. 7 and 8 . It might be significant that the $H_{0}=67 \mathrm{~km} \mathrm{~s}^{-1} \mathrm{Mpc}^{-1}$ case always has a lower $\chi^{2}$ than the $H_{0}=71 \mathrm{~km} \mathrm{~s}^{-1}$ $\mathrm{Mpc}^{-1}$ case, indicating perhaps that the median statistics value determined from a large fraction of the data is more robust - time will tell. In any case, a comparison of the entries in Table 1 shows that the results presented here are robust with respect to small changes in the value of $H_{0}$.

\footnotetext{
${ }^{5}$ The additional 130 measurements (an increase of $40 \%$ ) shift the median value to $H_{0}=68 \mathrm{~km} \mathrm{~s}^{-1} \mathrm{Mpc}^{-1}$ (using all 461 measurements); the small shift in the median after a $40 \%$ increase in the number of measurements considered is great tribute to its robustness. See Gott et al. (2001), Podariu et al. (2001), Avelino, Martins, \& Pinto (2002), and Chen \& Ratra (2003) for other cosmological applications of median statistics.
} 
Table 1. Goodness of Fit Numerical Values

\begin{tabular}{|c|c|c|c|c|c|c|c|c|}
\hline \multirow[b]{2}{*}{$\begin{array}{c}\text { function } \\
\text { (1) }\end{array}$} & \multicolumn{4}{|c|}{$H_{0}=71 \mathrm{~km} \mathrm{~s}^{-1} \mathrm{Mpc}^{-1}$} & \multicolumn{4}{|c|}{$H_{0}=67 \mathrm{~km} \mathrm{~s}^{-1} \mathrm{Mpc}^{-1}$} \\
\hline & $\begin{array}{l}\text { scale }^{\mathrm{a}} \\
(2)\end{array}$ & $\begin{array}{l}\chi_{\nu}^{2 \mathrm{~b}} \\
(3)\end{array}$ & $\begin{array}{c}\nu^{\mathrm{b}} \\
(4)\end{array}$ & $\begin{array}{c}\text { prob. }(\%)^{\mathrm{c}} \\
(5)\end{array}$ & $\begin{array}{l}\text { scale }^{\mathrm{a}} \\
(6)\end{array}$ & $\begin{array}{l}\chi_{\nu}^{2 \mathrm{~b}} \\
(7)\end{array}$ & $\begin{array}{c}\nu^{\mathrm{b}} \\
(8)\end{array}$ & $\begin{array}{c}\text { prob. }(\%)^{\mathrm{c}} \\
(9)\end{array}$ \\
\hline Gaussian & 1 & 19.8 & 20 & $<0.1$ & 1 & 15.0 & 20 & $<0.1$ \\
\hline Gaussian & 1.8 & 2.63 & 19 & $<0.1$ & 1.7 & 1.92 & 19 & 0.94 \\
\hline Cauchy & 1 & 1.42 & 20 & 9.9 & 1 & 1.10 & 20 & 35 \\
\hline Cauchy & 1.1 & 1.46 & 19 & 8.7 & 1.0 & 1.15 & 19 & 29 \\
\hline$n=2$ Student's $t$ & 1 & 2.58 & 19 & $<0.1$ & 1 & 1.56 & 19 & 5.7 \\
\hline$n=2$ Student's $t$ & 1.3 & 0.717 & 18 & 80 & 1.2 & 0.326 & 18 & 99.7 \\
\hline Double Exponential & 1 & 7.12 & 20 & $<0.1$ & 1 & 5.11 & 20 & $<0.1$ \\
\hline Double Exponential & 1.5 & 0.501 & 19 & 96 & 1.6 & 0.325 & 19 & 99.7 \\
\hline
\end{tabular}

a Scale (factor) $S=1$ corresponds to the case when $\left|N_{\sigma}\right|=1$ corresponds to one standard deviation for a Gaussian distribution, otherwise the width of the distribution is allowed to vary with the scale factor as $\chi^{2}$ is minimized.

${ }^{\mathrm{b}} \chi_{\nu}^{2}$ is the $\chi^{2}$ per degree of freedom, where $\nu$ is the number of degrees of freedom.

${ }^{c}$ Probability that a random sample of data points drawn from the assumed distribution yields a value of $\chi_{\nu}^{2}$ greater than or equal to the observed value for $\nu$ degrees of freedom. The computation assumes that the bins are uncorrelated, which is not necessarily true, so the probabilities should not be taken at face value but merely as qualitative indicatiors of goodness of fit. 
Table 2. $\left|N_{\sigma}\right|$ Limits

\begin{tabular}{|c|c|c|c|c|c|c|}
\hline \multirow[b]{2}{*}{$\begin{array}{c}\text { function } \\
\text { (1) }\end{array}$} & \multicolumn{3}{|c|}{$H_{0}=71 \mathrm{~km} \mathrm{~s}^{-1} \mathrm{Mpc}^{-1}$} & \multicolumn{3}{|c|}{$H_{0}=67 \mathrm{~km} \mathrm{~s}^{-1} \mathrm{Mpc}^{-1}$} \\
\hline & $\begin{array}{l}\text { scale }^{\mathrm{a}} \\
(2)\end{array}$ & $\begin{array}{c}68.3 \% \mathrm{~b} \\
(3)\end{array}$ & $\begin{array}{c}95.4 \% \mathrm{~b} \\
(4)\end{array}$ & $\begin{array}{l}\text { scale }^{\mathrm{a}} \\
(5)\end{array}$ & $\begin{array}{c}68.3 \% \mathrm{~b} \\
(6)\end{array}$ & $\begin{array}{c}95.4 \% \mathrm{~b} \\
(7)\end{array}$ \\
\hline Gaussian & 1 & 1.0 & 2.0 & 1 & 1.0 & 2.0 \\
\hline Gaussian & 1.8 & 1.8 & 3.6 & 1.7 & 1.7 & 3.4 \\
\hline Cauchy & 1 & 1.8 & 14 & 1 & 1.8 & 14 \\
\hline Cauchy & 1.1 & 2.0 & 15 & 1.0 & 1.8 & 14 \\
\hline$n=2$ Student's $t$ & 1 & 1.3 & 4.5 & 1 & 1.3 & 4.5 \\
\hline$n=2$ Student's $t$ & 1.3 & 1.7 & 5.9 & 1.2 & 1.6 & 5.4 \\
\hline Double Exponential & 1 & 1.2 & 3.1 & 1 & 1.2 & 3.1 \\
\hline Double Exponential & 1.5 & 1.7 & 4.6 & 1.6 & 1.8 & 4.9 \\
\hline Observed & & 1.9 & 7.0 & & 1.7 & 7.5 \\
\hline
\end{tabular}

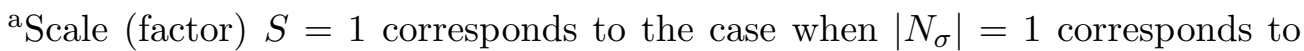
one standard deviation for a Gaussian distribution, otherwise the width of the distribution is allowed to vary with the scale factor as $\chi^{2}$ is minimized. For a given distribution the derived limits depend only on the value of $S$.

${ }^{\mathrm{b}}$ Upper $\left|N_{\sigma}\right|$ limits that include $68.3 \%$ and $95.4 \%$ of the probability. 
Table 3. Expected Fraction of Data Points with $\left|N_{\sigma}\right| \leq 1$ and $\left|N_{\sigma}\right| \leq 2$

\begin{tabular}{|c|c|c|c|c|c|c|}
\hline \multirow[b]{2}{*}{$\begin{array}{c}\text { function } \\
\text { (1) }\end{array}$} & \multicolumn{3}{|c|}{$H_{0}=71 \mathrm{~km} \mathrm{~s}^{-1} \mathrm{Mpc}^{-1}$} & \multicolumn{3}{|c|}{$H_{0}=67 \mathrm{~km} \mathrm{~s}^{-1} \mathrm{Mpc}^{-1}$} \\
\hline & $\begin{array}{c}\text { scale }^{\mathrm{a}} \\
(2)\end{array}$ & $\begin{array}{c}\left|N_{\sigma}\right| \leq 1^{\mathrm{b}} \\
(3)\end{array}$ & $\begin{array}{c}\left|N_{\sigma}\right| \leq 2^{\mathrm{b}} \\
\quad(4)\end{array}$ & $\begin{array}{l}\text { scale }^{\mathrm{a}} \\
(5)\end{array}$ & $\begin{array}{c}\left|N_{\sigma}\right| \leq 1^{\mathrm{b}} \\
\quad(6)\end{array}$ & $\begin{array}{c}\left|N_{\sigma}\right| \leq 2^{\mathrm{b}} \\
\quad(7)\end{array}$ \\
\hline Gaussian & 1 & 0.68 & 0.95 & 1 & 0.68 & 0.95 \\
\hline Gaussian & 1.8 & 0.42 & 0.73 & 1.7 & 0.44 & 0.76 \\
\hline Cauchy & 1 & 0.50 & 0.71 & 1 & 0.50 & 0.71 \\
\hline Cauchy & 1.1 & 0.47 & 0.68 & 1.0 & 0.50 & 0.71 \\
\hline$n=2$ Student's $t$ & 1 & 0.58 & 0.82 & 1 & 0.58 & 0.82 \\
\hline$n=2$ Student's $t$ & 1.3 & 0.48 & 0.74 & 1.2 & 0.51 & 0.76 \\
\hline Double Exponential & 1 & 0.63 & 0.87 & 1 & 0.63 & 0.87 \\
\hline Double Exponential & 1.5 & 0.49 & 0.74 & 1.6 & 0.47 & 0.71 \\
\hline Observed & & 0.48 & 0.69 & & 0.51 & 0.72 \\
\hline
\end{tabular}

a Scale (factor) $S=1$ corresponds to the case when $\left|N_{\sigma}\right|=1$ corresponds to one standard deviation for a Gaussian distribution, otherwise the width of the distribution is allowed to vary with the scale factor as $\chi^{2}$ is minimized. For a given distribution the derived limits depend only on the value of $S$.

${ }^{\mathrm{b}}$ Fraction of data points with $\left|N_{\sigma}\right| \leq 1$ or $\left|N_{\sigma}\right| \leq 2$. 


\section{REFERENCES}

Avelino, P. P., Martins, C. J. A. P., \& Pinto, P. 2002, ApJ, 575, 989

Bennett, C. L., et al. 2003, ApJ, in press, astro-ph/0302207

Chen, G., \& Ratra, B. 2003, PASP, in press, astro-ph/0302002

Eadie, W. T., Drijard, D., James, F. E., Roos, M., \& Sadoulet, B. 1971, Statistical Methods in Experimental Physics (Amsterdam: North-Holland), p. 84

Freedman, W. L., et al. 2001, ApJ, 553, 47

Ganga, K., Ratra, B., \& Sugiyama, N. 1996, ApJ, 461, L61

Gott, J. R., Vogeley, M. S., Podariu, S., \& Ratra, B. 2001, ApJ, 549, 1

Peebles, P. J. E., \& Ratra, B. 2003, Rev. Mod. Phys., 75, 559

Podariu, S., Souradeep, T., Gott, J. R., Ratra, B., \& Vogeley, M. S. 2001, ApJ, 559, 9

Spergel, D. N., et al. 2003, ApJ, in press, astro-ph/0302209 

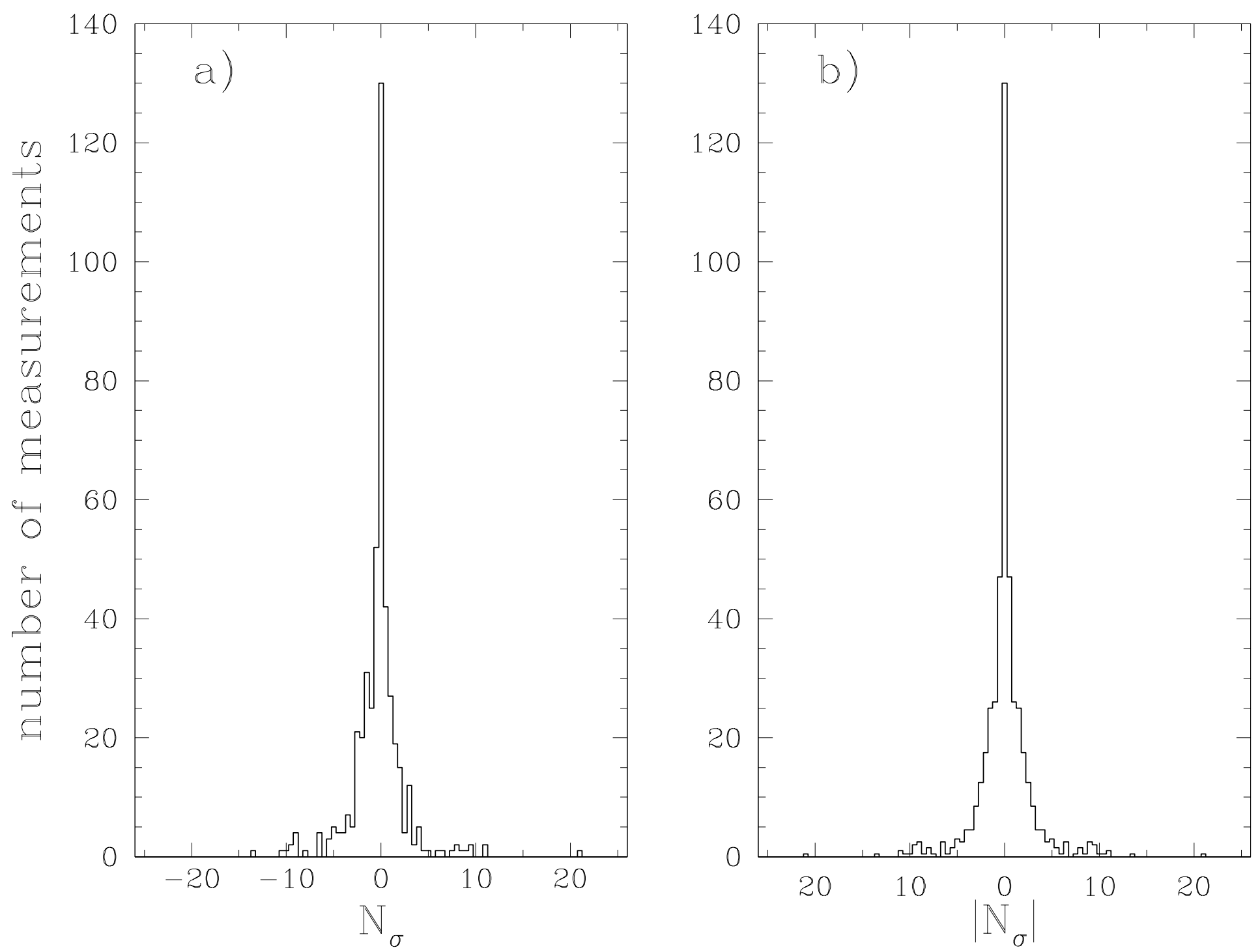

Fig. 1.- Number of measurements (in half standard deviation bins) away from the central value of $H_{0}=71 \mathrm{~km} \mathrm{~s}^{-1} \mathrm{Mpc}^{-1}$ estimated by the WMAP collaboration. Left panel $a$ ) shows the sign of the deviation while right panel $b$ ) shows only the magnitude of the deviation. In panel $a$ ) bins with positive (negative) $N_{\sigma}$ correspond to measurements where $H_{0}$ is measured to be higher (lower) than $71 \mathrm{~km} \mathrm{~s}^{-1} \mathrm{Mpc}^{-1}$. 

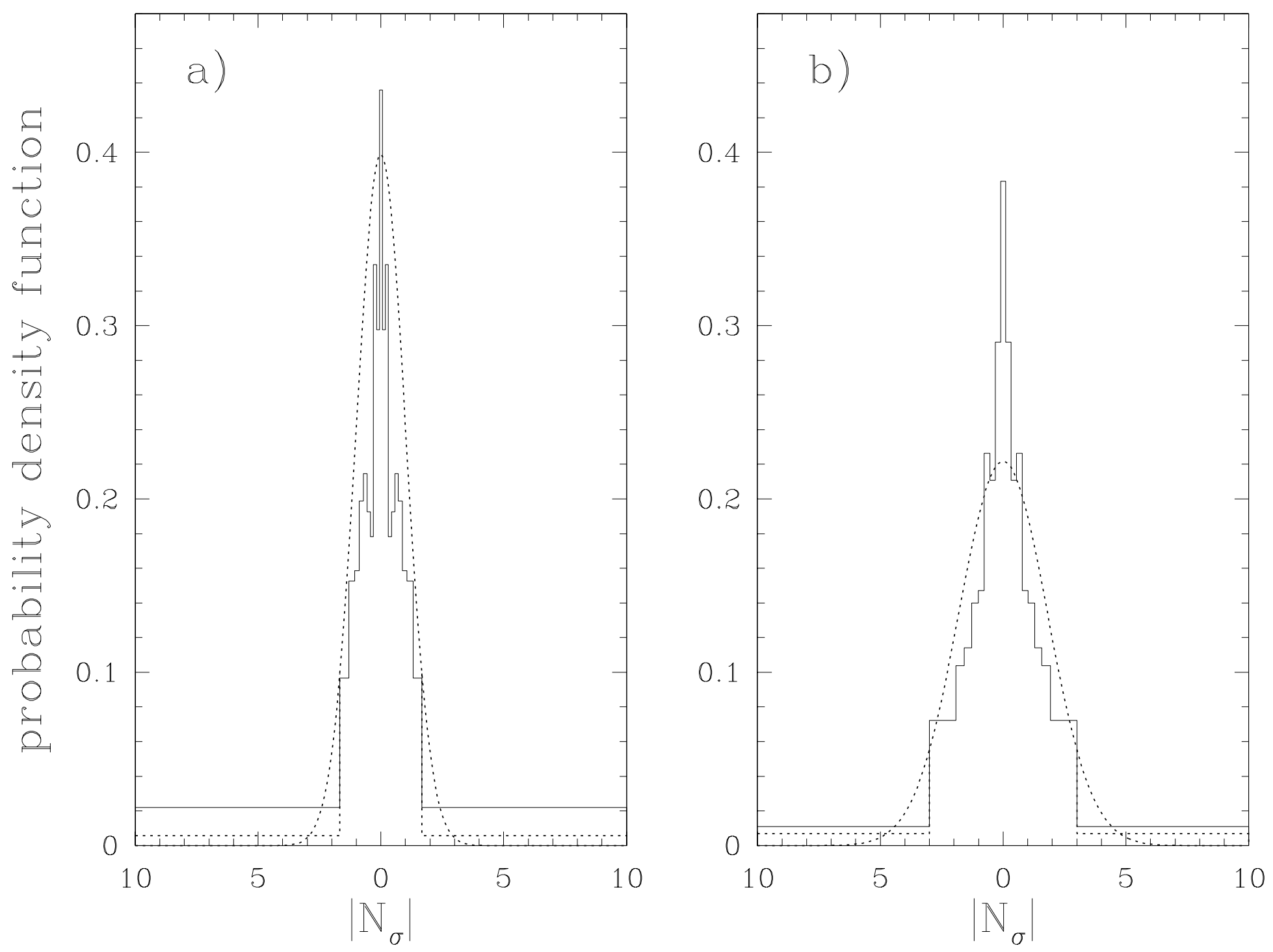

Fig. 2.- Binned data (solid lines) and best-fit Gaussian probability distribution functions (dotted lines) for $H_{0}=71 \mathrm{~km} \mathrm{~s}^{-1} \mathrm{Mpc}^{-1}$ estimated by the WMAP collaboration, all normalized to unit area. The binning and therefore the data histogram shapes depend on the assumed probability distribution function (see text). Left panel $a$ ) shows a Gaussian distribution with scale factor $S=1$ such that $\left|N_{\sigma}\right|=1$ corresponds to one standard deviation; right panel $b$ ) allows $S$ to vary as $\chi^{2}$ is minimized and the best fit value of $S=2.0$ is shown. For ease of visualization, the extreme ends of the left- and right-most bins (solid lines) have been brought in from $\left|N_{\sigma}\right|=\infty$ to $\left|N_{\sigma}\right|=10$, with the heights adjusted to ensure that the probabilities in the bins are unchanged. The dotted horizontal and vertical lines demarcate the predicted probability for these last bins for the assumed Gaussian distribution. 

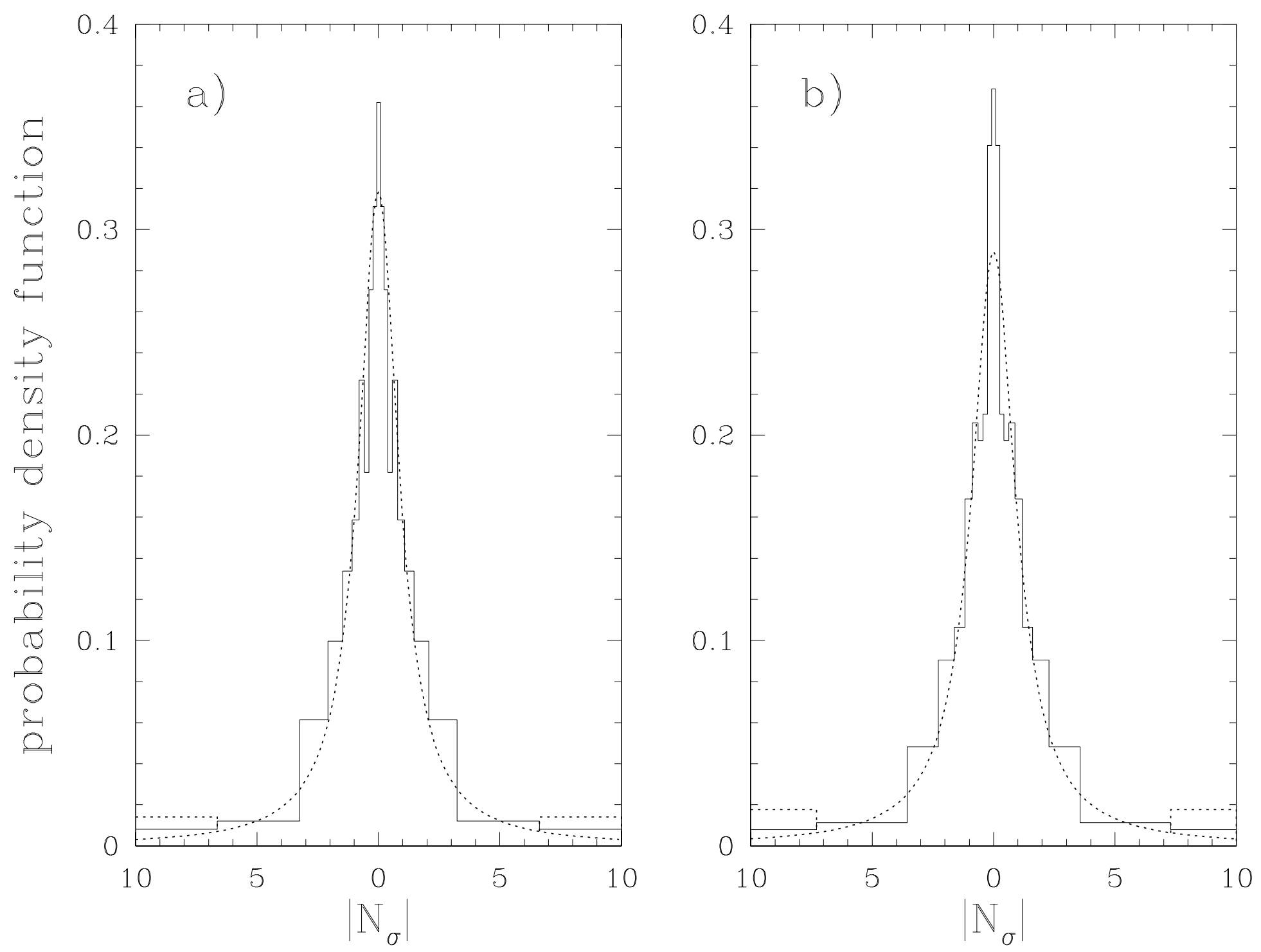

Fig. 3.- Binned data (solid lines) and best-fit Cauchy probability distribution functions (dotted lines) for $H_{0}=71 \mathrm{~km} \mathrm{~s}^{-1} \mathrm{Mpc}^{-1}$ estimated by the WMAP collaboration, all normalized to unit area. See Fig. 2 caption for more details. Left panel $a$ ) shows a Cauchy distribution with scale factor $S=1$; right panel $b$ ) allows $S$ to vary as $\chi^{2}$ is minimized and the best fit value of $S=1.1$ is shown. The dotted horizontal and vertical lines demarcate the predicted probability for the last bins for the assumed Cauchy distribution. 

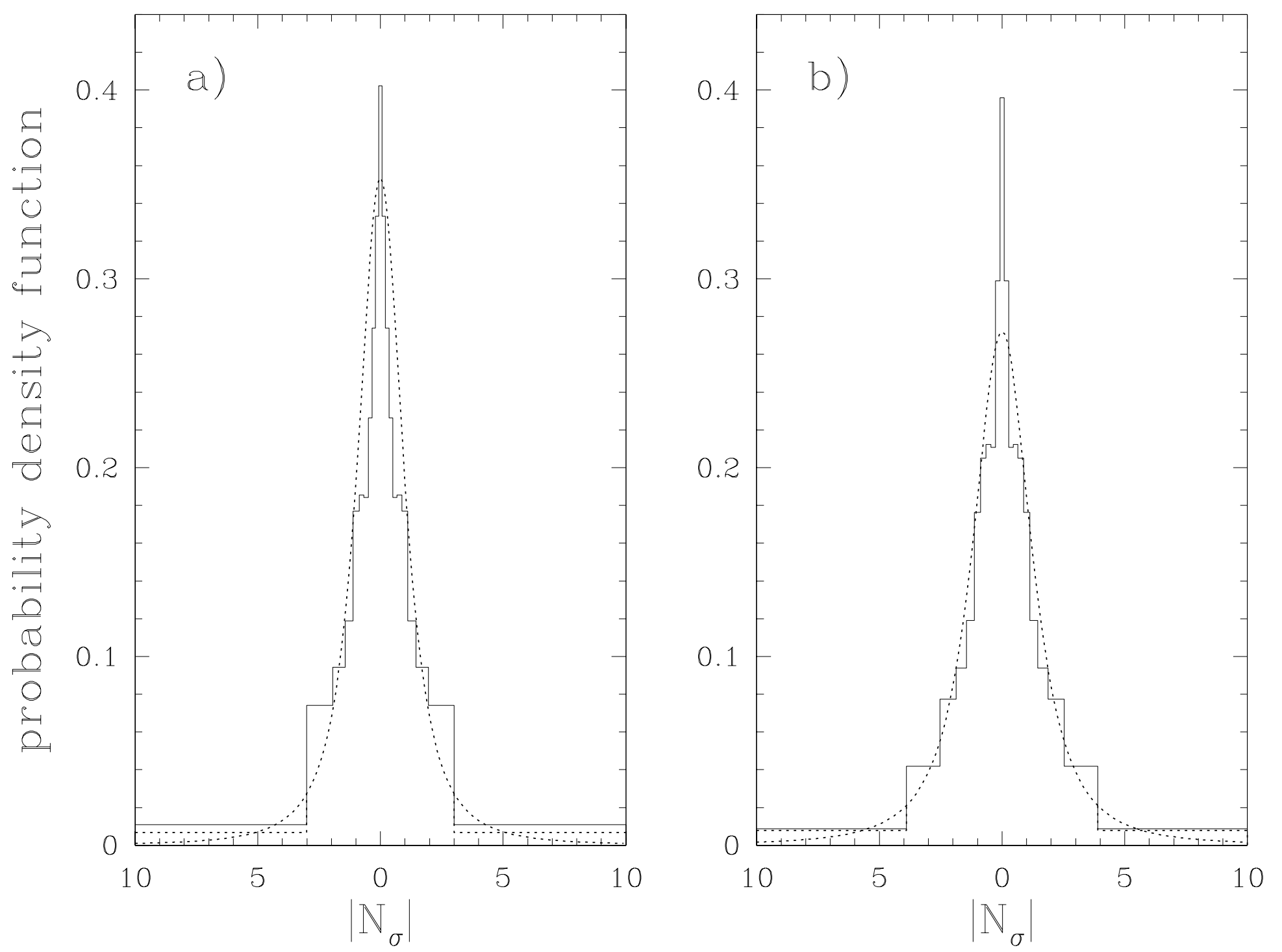

Fig. 4. - Binned data (solid lines) and best-fit $n=2$ Student's $t$ probability distribution functions (dotted lines) for $H_{0}=71 \mathrm{~km} \mathrm{~s}^{-1} \mathrm{Mpc}^{-1}$ estimated by the WMAP collaboration, all normalized to unit area. See Fig. 2 caption for more details. Left panel $a$ ) shows a distribution with scale factor $S=1$; right panel $b$ ) allows $S$ to vary as $\chi^{2}$ is minimized and the best fit value of $S=1.3$ is shown. The dotted horizontal and vertical lines demarcate the predicted probability for the last bins for the assumed distribution. 

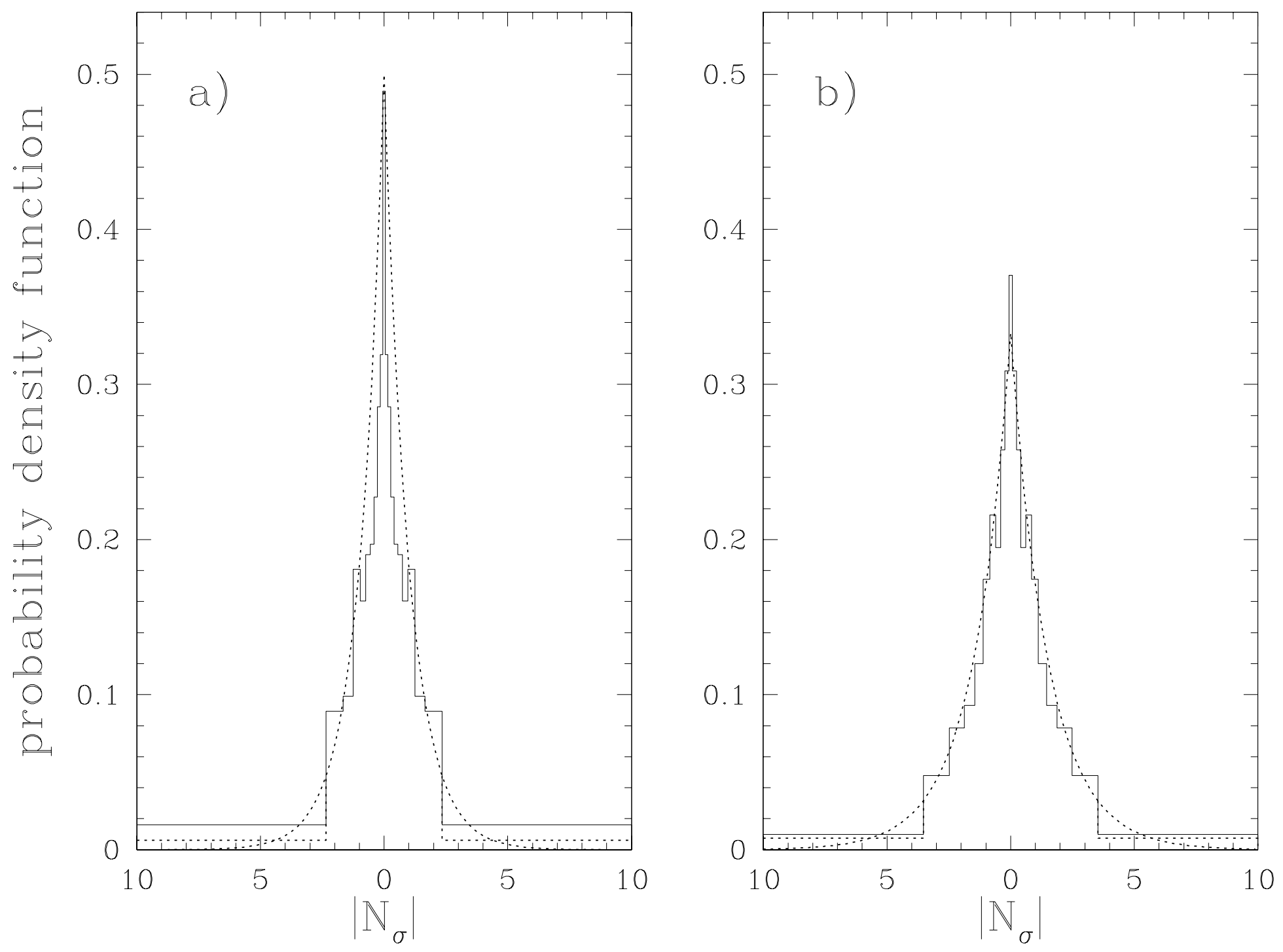

Fig. 5.- Binned data (solid lines) and best-fit double exponential probability distribution functions (dotted lines) for $H_{0}=71 \mathrm{~km} \mathrm{~s}^{-1} \mathrm{Mpc}^{-1}$ estimated by the WMAP collaboration, all normalized to unit area. See Fig. 2 caption for more details. Left panel $a$ ) shows a distribution with scale factor $S=1$; right panel $b$ ) allows $S$ to vary as $\chi^{2}$ is minimized and the best fit value of $S=1.5$ is shown. The dotted horizontal and vertical lines demarcate the predicted probability for the last bins for the assumed double exponential distribution. 

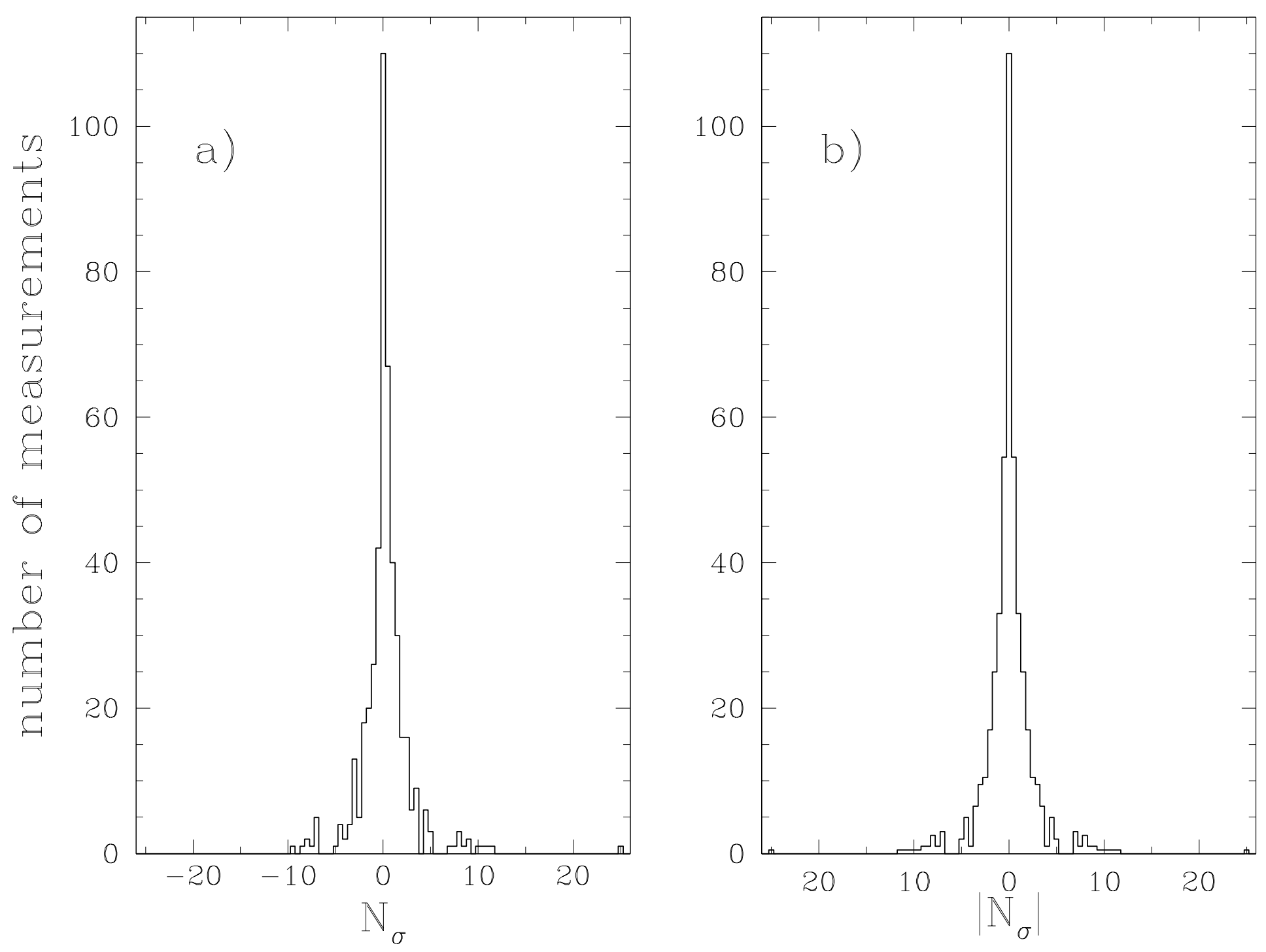

Fig. 6. - Number of measurements (in half standard deviation bins) away from the central value of $H_{0}=67 \mathrm{~km} \mathrm{~s}^{-1} \mathrm{Mpc}^{-1}$ estimated using median statistics on a major subset of the 461 measurements used in this paper. Left panel $a$ ) shows the sign of the deviation while right panel $b$ ) shows only the magnitude of the deviation. 

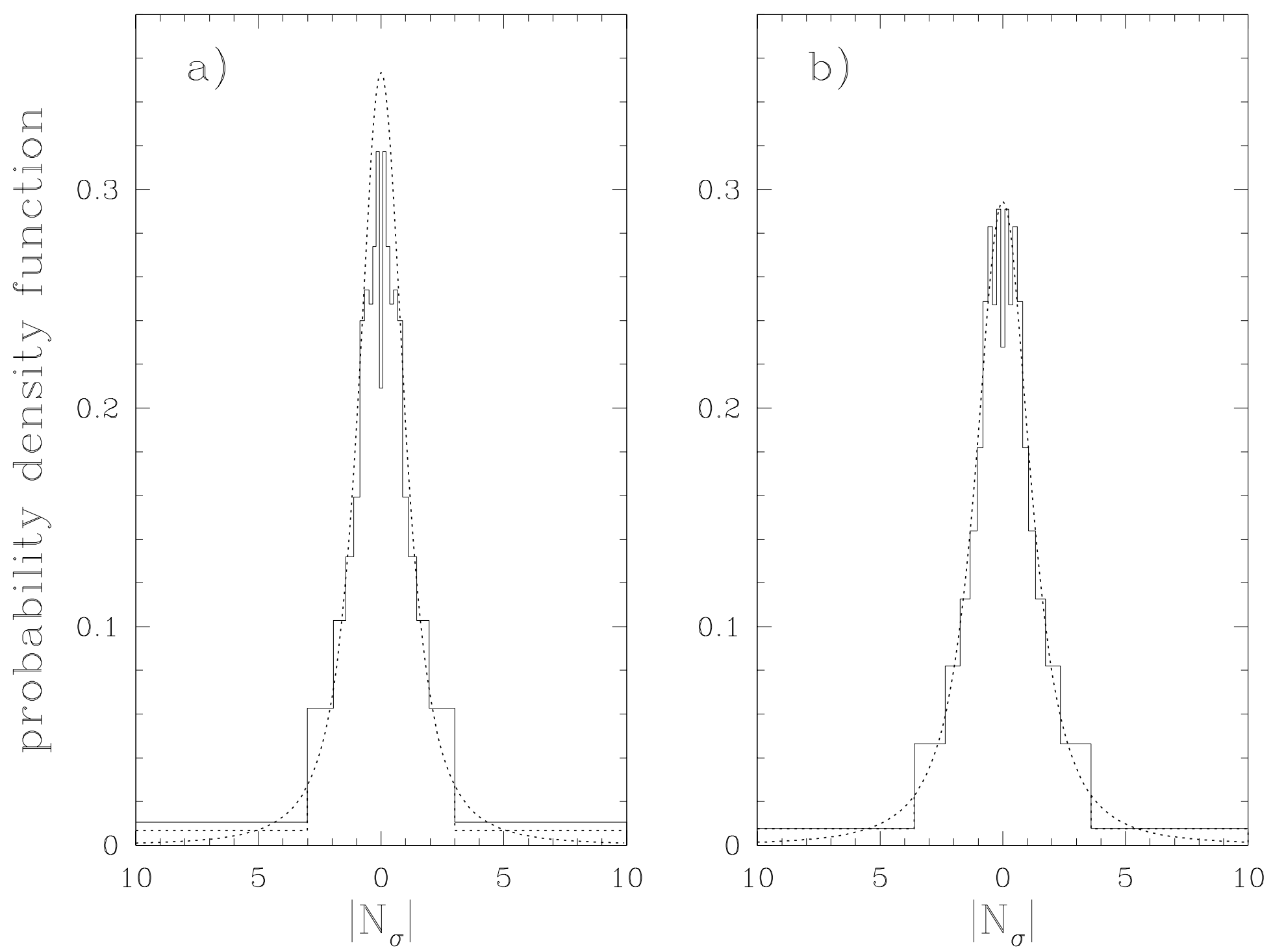

Fig. 7. - Binned data (solid lines) and best-fit $n=2$ Student's $t$ probability distribution functions (dotted lines) for $H_{0}=67 \mathrm{~km} \mathrm{~s}^{-1} \mathrm{Mpc}^{-1}$ estimated using median statistics, all normalized to unit area. See Fig. 2 caption for more details. Left panel $a$ ) shows a distribution with scale factor $S=1$; right panel $b$ ) allows $S$ to vary as $\chi^{2}$ is minimized and the best fit value of $S=1.2$ is shown. The dotted horizontal and vertical lines demarcate the predicted probability for the last bins for the assumed distribution. 

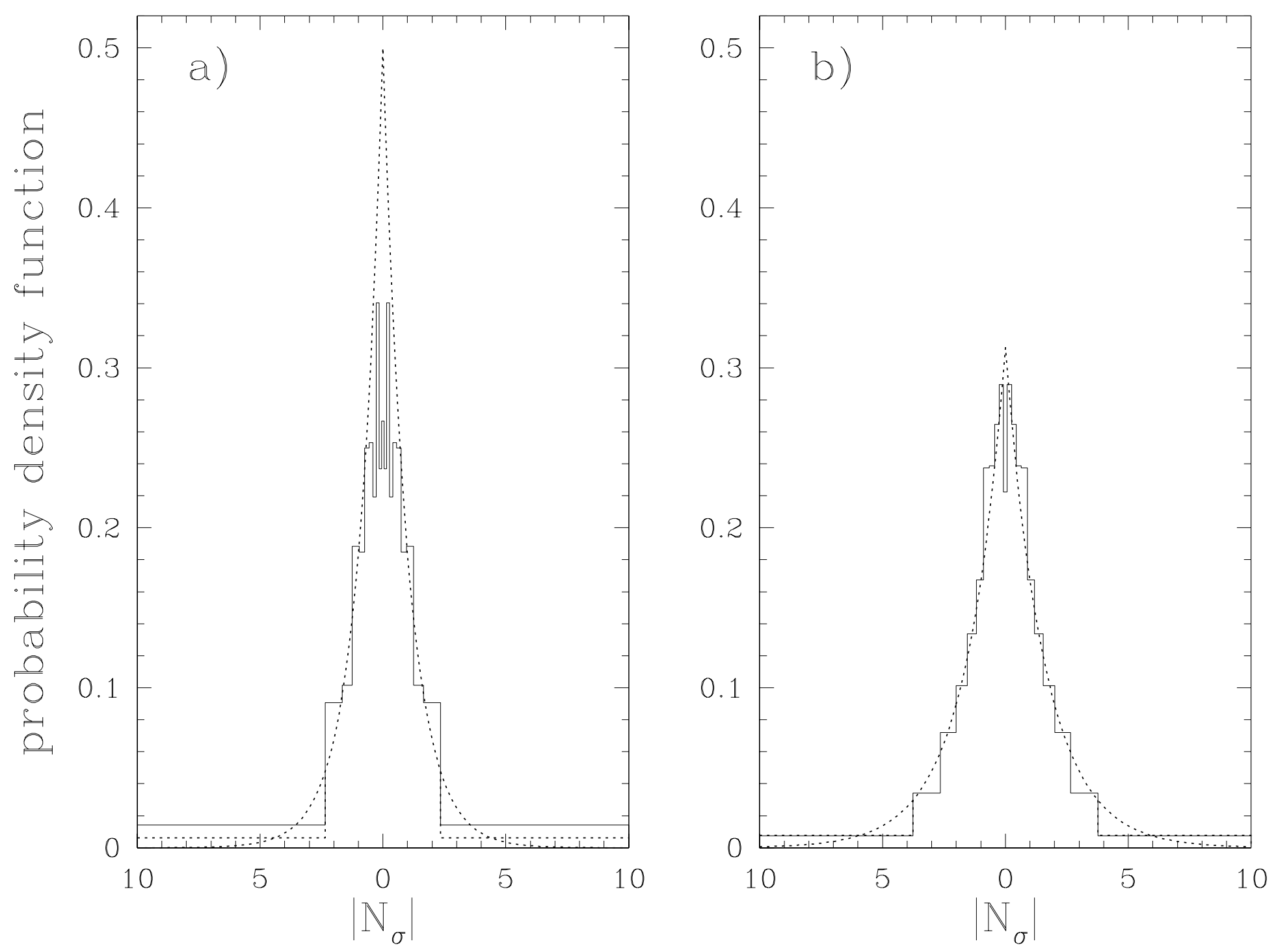

Fig. 8.- Binned data (solid lines) and best-fit double exponential probability distribution functions (dotted lines) for $H_{0}=67 \mathrm{~km} \mathrm{~s}^{-1} \mathrm{Mpc}^{-1}$ estimated using median statistics, all normalized to unit area. See Fig. 2 caption for more details. Left panel $a$ ) shows a distribution with scale factor $S=1$; right panel $b$ ) allows $S$ to vary as $\chi^{2}$ is minimized and the best fit value of $S=1.5$ is shown. The dotted horizontal and vertical lines demarcate the predicted probability for the last bins for the assumed distribution. 